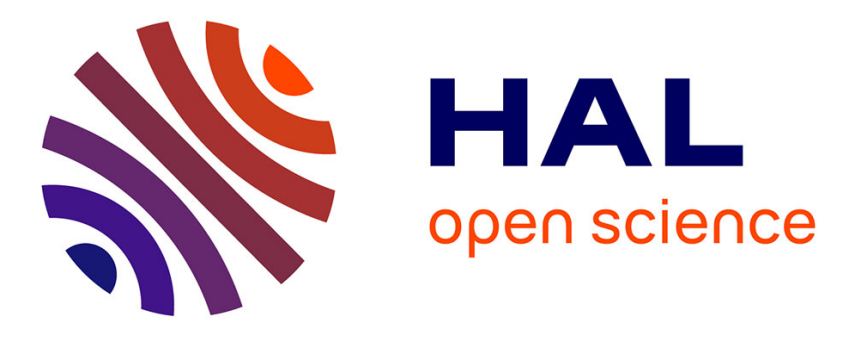

\title{
Sur la forme de la boule unité de la norme stable unidimensionnelle
}

\author{
Ivan K. Babenko, Florent N. Balacheff
}

\section{To cite this version:}

Ivan K. Babenko, Florent N. Balacheff. Sur la forme de la boule unité de la norme stable unidimensionnelle. Manuscripta mathematica, 2006, 119 (3), pp.347-358. 10.1007/s00229-005-0622-x . hal-00004322

\section{HAL Id: hal-00004322 \\ https://hal.science/hal-00004322}

Submitted on 22 Feb 2005

HAL is a multi-disciplinary open access archive for the deposit and dissemination of scientific research documents, whether they are published or not. The documents may come from teaching and research institutions in France or abroad, or from public or private research centers.
L'archive ouverte pluridisciplinaire HAL, est destinée au dépôt et à la diffusion de documents scientifiques de niveau recherche, publiés ou non, émanant des établissements d'enseignement et de recherche français ou étrangers, des laboratoires publics ou privés. 


\title{
Sur la forme de la boule unité de la norme stable unidimensionnelle
}

\author{
Ivan BABENKO ${ }^{*}$ Florent BALACHEFF ${ }^{\dagger}$
}

22 février 2005

\begin{abstract}
Résumé
Pour un polyèdre riemannien, nous étudions les formes apparaissant comme boule unité de la norme stable unidimensionnelle (boule stable). Dans le cas d'un polyèdre riemannien unidimensionnel (graphe), nous montrons que la boule stable est un polytope dont les sommets sont complètement décrits par la combinatoire du graphe. Nous étudions ensuite les formes réalisables comme boule stable de variétés riemanniennes de dimension plus grande que trois. Nous montrons que, pour une variété riemannienne $(M, g)$ fixée, une large classe de polytopes peut apparaître comme boule stable d'une métrique dans la classe conforme de $g$. Nous utilisons pour cela une technique polyèdrale.
\end{abstract}

Mathematics Subject Classification (2000) : 05C38, 52B05, 53C20, 53C23.

Mots clefs : variété riemannienne, polyèdre riemannien, norme stable, boule unité.

\section{Introduction}

\subsection{Enoncé des résultats}

Pour un polyèdre fini riemannien $(M, g)$ de classe $C^{0}$, l'homologie réelle possède une norme naturelle appelée masse ou norme stable (voir [6], [7]). Cette norme est particulièrement intéressante pour l'homologie de dimension 1 : elle contrôle le comportement à l'infini de la géométrie relevée sur le revêtement homologique correspondant. Nous rappelons ici la plus géométrique des définitions en renvoyant le lecteur à [6] pour les différentes approches et la démonstration des équivalences entre celles-ci.

Définition 1 Soit $v \in H_{1}(M, \mathbb{R})$ une classe entière. On pose

$$
\|v\|_{g}=\lim _{n \rightarrow+\infty} \frac{L_{g}\left(\gamma_{n}\right)}{n}
$$

*UMR 5149, Institut de Mathématiques et de Modélisation de Montpellier, Université Montpellier II Case Courrier 051 - Place Eugène Bataillon 34095 Montpellier CEDEX 5, France e-mail: babenko@math.univ-montp2.fr

${ }^{\dagger}$ UMR 5149, Institut de Mathématiques et de Modélisation de Montpellier, Université Montpellier II Case Courrier 051 - Place Eugène Bataillon 34095 Montpellier CEDEX 5, France e-mail: balachef@math.univ-montp2.fr 
où $\gamma_{n}$ est la plus petite courbe fermée réalisant la classe $n . v$ et $L_{g}$ désigne la longueur respectivement à $\mathrm{g}$. Cela définit par continuité une norme sur $H_{1}(M, \mathbb{R})$ appelée norme stable. On pose alors :

$$
\mathcal{B}_{s t}(g)=\left\{u \in H_{1}(M, \mathbb{R}) \mid\|u\|_{g} \leq 1\right\} .
$$

Un point $s$ de $H_{1}(M, \mathbb{R})$ est dit de direction rationnel si il existe une classe entière $v$ telle que $s \in\langle v\rangle$ la droite engendrée par $v$. Dans le cas contraire, le point est dit de direction irrationnelle.

Les variétés riemanniennes et les polyèdres unidimensionnels (ou graphes) constituent deux sous-classes naturelles de polyèdres riemanniens pour lesquelles nous allons étudier les formes réalisables comme boule unité de la norme stable associée à une métrique.

Nous commençons par étudier la classe des graphes finis. La métrique naturelle d'un graphe pondéré est celle induite par la métrique sur ses arêtes vues comme intervalles de longueur donnée par le poids. Comme nous le démontrons dans la section 2.1, la boule unité de la norme stable d'un graphe pondéré est toujours un polytope. On peut décrire complètement la forme de ce polytope en terme de la combinatoire du graphe. Pour cela, étant donné un graphe $G$, un chemin fermé orienté parcourant tous ses sommets une seule fois est dit circuit simple orienté. Deux circuits simples qui coïncident géométriquement et sont de même orientation, mais qui diffèrent par leur point initial sont identifiés. On considère l'espace vectoriel $\mathcal{C}(G, \mathbb{R})$ engendré par les arêtes de $G$ après le choix d'une orientation arbitraire. A chaque circuit simple orienté est associé un vecteur de $\mathcal{C}(G, \mathbb{R})$ - la somme (avec les signes nécessaires) des arêtes formant ce circuit. Les vecteurs ainsi construits sont de nouveau appelés circuits simples orientés. Notons qu'à un circuit géométrique simple correspond deux circuits simples orientés.

Théorème $\mathbf{A}$ Soit $(G, w)$ un graphe pondéré. On note $\left\{C_{j}\right\}_{j \in J}$ l'ensemble de ses circuits simples orientés.

Alors la boule unité de la norme stable pour la métrique $w$ dans $H_{1}(G, \mathbb{R})$ coïncide avec l'enveloppe convexe dans $\mathcal{C}(G, \mathbb{R})$ des vecteurs $\left\{C_{j} /\left\|C_{j}\right\|_{w}\right\}_{j \in J}$.

A premier nombre de Betti $b$ fixé, le nombre de circuits simples orientés d'un graphe admet une majoration évidente :

Corollaire A Soit $(G, w)$ un graphe pondéré de premier nombre de Betti b. Alors la boule unité de sa norme stable est un polytope b-dimensionnel dont le nombre de sommets est majoré par $2\left(2^{b}-1\right)$.

Etant fixée une variété fermée $M$, nous allons nous intéresser maintenant aux normes qui peuvent être réalisées comme norme stable d'une métrique lisse (i.e de classe $\left.C^{\infty}\right)$.

Théorème $\mathbf{B}$ Soit $(M, g)$ une variété fermée riemannienne lisse de dimension $m \geq 3$ et de premier nombre de Betti $b \geq 1$. On considère un polytope fini convexe $K$ de $H_{1}(M, \mathbb{R})$, à symétrie centrale, tel que son intérieur soit non vide et les directions de ses sommets soient rationnelles. Alors il existe une métrique $g^{\prime}$ lisse sur $M$ conforme à $g$ telle que

$$
\mathcal{B}_{s t}\left(g^{\prime}\right)=K
$$

On en déduit immédiatement le corollaire suivant : 
Corollaire B Soient $(M, g)$ une variété riemannienne de dimension $m \geq 3$ et de premier nombre de Betti $b \geq 1$ et $\|\cdot\|$ une norme sur l'espace vectoriel $H_{1}(M, \mathbb{R})$. Pour tout $\varepsilon>0$, il existe une métrique $g(\varepsilon)$ conforme à $g$ telle que

$$
\|\cdot\| \leq\|\cdot\|_{g(\varepsilon)} \leq(1+\varepsilon)\|\cdot\| .
$$

\subsection{Historique}

Nous récapitulons ici l'ensemble des résultats traitant de la question suivante : étant fixée une variété $M$, quelles normes peuvent être réalisées comme norme stable associée à une métrique $g$ ?

Cas du tore bidimensionnel. M.Morse [12] a tout d'abord remarqué que la norme stable du tore bidimensionnel $\mathbb{T}^{2}$ associée à une métrique lisse est strictement convexe. V. Bangert [4] a ensuite montré le résultat suivant :

Théorème 1 Soit $\left(\mathbb{T}^{2}, g\right)$ un tore riemannien lisse. La norme stable est différentiable dans les points de direction irrationnelle et est différentiable dans les points de direction rationnelle si et seulement si le tore est feuilletté par les géodésiques minimisantes représentant l'élément correspondant de $H_{1}\left(\mathbb{T}^{2}, \mathbb{Z}\right)$.

Cas des surfaces. On note $\Sigma_{h}$ la surface fermée orientable de genre $h$. On se fixe une métrique $g$ lisse. Un sous-espace affine support de $\mathcal{B}_{s t}(g)$ est un sousespace affine $H$ tel que $H \cap \mathcal{B}_{s t}(g)=H \cap \partial \mathcal{B}_{s t}(g)$. On dit d'un sous-ensemble $F$ de $\partial \mathcal{B}_{s t}(g)$ qu'elle est une face plate si il existe un sous-espace affine support $H$ tel que $F=H \cap \partial \mathcal{B}_{s t}(g)$. La dimension de $F$ est alors définie comme la dimension de l'espace affine $A$ qu'elle engendre et l'intérieur de $F$ est son intérieur dans $A$. Enfin une face plate est rationnelle si elle cö̈ncide avec l'enveloppe convexe de points de $\partial \mathcal{B}_{s t}(g)$ de direction rationnelle. Dans [9], D.Massart montre que l'ensemble des faces contenant un point de $\partial \mathcal{B}_{s t}(g)$ dans leur intérieur peut être ordonné par inclusion et admet un maximum unique. Il montre également le résultat suivant :

Théorème 2 Soit $g$ une métrique lisse sur $\Sigma_{h}$ avec $h \geq 2$.

1. La dimension d'une face plate est majorée par $h-1$.

2. Tout point de direction rationnelle est contenu dans une face plate rationnelle de dimension $h-1$.

3. En un point $v$ de $\partial \mathcal{B}_{s t}(g)$ de direction rationnelle, la norme stable est différentiable seulement dans les directions tangentes à $F$, où $F$ est la face plate rationnelle maximale contenant $v$ dans son intérieur.

On en déduit immédiatement que la norme stable d'une surface $\left(\Sigma_{h}, g\right)$ de genre $h \geq 2$ n'est donc ni lisse, ni strictement convexe, ni définie par un polytope. Remarquons également qu'il existe une infinité de points exposés de direction rationnelle pour de telles surfaces (un point est dit exposé si la face rationnelle maximale le contenant dans son intérieur est réduite à ce point) (voir 10]).

Dans [1], D.Massart relie la dérivabilité de la norme stable en une classe d'homologie au degré d'irrationalité de cette classe. Plus précisément, une classe d'homologie $v$ est dite $k$-irrationnelle si $k$ est la dimension du plus petit sous-espace de $H_{1}\left(\Sigma_{h}, \mathbb{R}\right)$ engendré par des classes entières et contenant $v$. 
Théorème 3 Soit $g$ une métrique lisse sur $\Sigma_{h}$ avec $h \geq 1$. En une classe $v$ d'homologie $k$-irrationnelle, la norme stable est différentiable dans au moins $k-1$ directions non radiales.

Cas des dimensions supérieures. G.A.Hedlund a construit pour le tore de dimension 3 une métrique Riemannienne [8] pour laquelle la norme stable est donnée par un octaèdre régulier. Cet exemple est fondamental, puisqu'il montre que la situation en dimension plus grande que 3 est radicalement différente de celle en dimension 2 : les polytopes peuvent apparaître comme boule unité d'une norme stable associée à une métrique.

D'autre part, D.Burago, S.Ivanov et B.Kleiner [5] ont démontré le résultat suivant, qui généralise le résultat de V.Bangert :

Théorème 4 Soit $\left(\mathbb{T}^{n}, g\right)$ un tore riemannien de classe $C^{3}$ de dimension $n \geq 2$. Soit $v$ un point de direction irrationnelle. Alors la norme stable est différentiable en $v$ dans au moins une direction non radiale.

Dans ce même papier, les auteurs montrent que, pour tout entier $k$, il existe un entier $n$ et une métrique de classe $C^{k}$ sur $\mathbb{T}^{n}$ telle que pour presque tous les points de direction irrationnelle $v$, la norme stable soit non différentiable en $v$. Cela montre qu'une généralisation complète du théorème de V.Bangert concernant les directions irrationnelles est impossible.

Les auteurs expriment leurs remerciements à D.Massart pour d'instructives et de stimulantes conversations.

Le papier est organisé de la manière suivante : les sections 2 et 3 correspondent respectivement aux démonstrations des théorèmes $\mathbf{A}$ et $\mathbf{B}$.

\section{Norme stable des graphes}

\subsection{Rappels sur les graphes}

Commençons par quelques définitions. Un graphe pondéré est une paire $(G, w)$ où $G=(V, E)$ est un multigraphe fini connexe non orienté et $w$ est une fonction poids sur les arêtes $w: E \rightarrow \mathbb{R}_{+}$. Un multigraphe désigne un graphe dans lequel on autorise les arêtes multiples et les boucles. On appelle $w(e)$ le poids d'une arête. Tout graphe est naturellement identifié à un graphe pondéré dans lequel le poids de chaque arête vaut 1 .

On se fixe pour la suite un graphe pondéré $(G, w)$ de premier nombre de Betti $b$. On choisit pour chaque arête une orientation arbitraire, notons ces arêtes $\left\{e_{i}\right\}_{i=1}^{k}$. L'espace vectoriel $\mathcal{C}(G, \mathbb{R})$ engendré par les arêtes orientées $\left\{e_{i}\right\}_{i=1}^{k}$ coïncide avec l'espace des chaînes simpliciales du complexe simplicial $G$ :

$$
\mathcal{C}(G, \mathbb{R})=\left\{\sum_{i=1}^{k} a_{i} \cdot e_{i} \mid a_{i} \in \mathbb{R} \text { pour } i=1, \ldots, k\right\}
$$

Comme les graphes n'ont pas de cellule pour les dimensions plus grandes que 2, l'homologie de $G$ de dimension 1 à coefficients réels $H_{1}(G, \mathbb{R})$ est plongée naturellement dans $\mathcal{C}(G)$ comme un sous-espace vectoriel de dimension $b$. L'homologie de $G$ 
de dimension 1 à coefficients entiers $H_{1}(G, \mathbb{Z})$, en l'absence de torsion dans ce cadre unidimensionnel, constitue un réseau du sous-espace $H_{1}(G, \mathbb{R})$ (comparer avec [2]).

Pour $u=\sum_{i=1}^{k} u_{i} . e_{i} \in \mathcal{C}(G, \mathbb{R})$, on note

$$
|u|_{w, 1}=\sum_{i=1}^{k} w_{i}\left|u_{i}\right|
$$

où $w_{i}=w\left(e_{i}\right)$ pour $i=1, \ldots, k$. On voit facilement que cette norme cö̈ncide avec la norme stable $\|\cdot\|_{w}$ sur $H_{1}(G, \mathbb{R})$. En effet, la norme stable de $v \in H_{1}(G, \mathbb{R})$ est donnée par la formule

$$
\|v\|_{w}=\inf \left\{\sum_{i=1}^{s}\left|\alpha_{i}\right| w\left(\sigma_{i}\right) \mid v=\sum_{i=1}^{s} \alpha_{i}\left[\sigma_{i}\right], \alpha_{i} \in \mathbb{R} \text { et } \sigma_{i} \in E\right\} .
$$

Cette définition est équivalente à la définition 1 (voir [6]).

La boule stable $\mathcal{B}_{s t}(G, w)$ est donc l'intersection de la boule unité de la norme $|\cdot|_{w, 1}$ dans l'espace des arêtes $\mathcal{C}(G, \mathbb{R})$ avec le sous-espace vectoriel de dimension $b$ image par le plongement naturel de l'homologie réelle de $G$ de dimension 1.

\subsection{Démonstration du théorème $\mathrm{A}$}

La démonstration du théorème $\mathbf{A}$ est une conséquence immédiate des lemmes suivants.

Lemme A1 Tout circuit simple orienté de $G$, identifié au vecteur correspondant de $\mathcal{C}(G, \mathbb{R})$, est proportionnel à un sommet de $\mathcal{B}_{s t}(G, w)$. Le facteur de proportionnalité est exactement la longueur de ce circuit.

Démonstration. Notons $B_{w, 1}$ la boule unité pour la norme $(2.1)$ dans $\mathcal{C}(G, \mathbb{R})$. Comme

$$
\mathcal{B}_{s t}(G, w)=B_{w, 1} \bigcap H_{1}(G, \mathbb{R}),
$$

les sommets de $\mathcal{B}_{s t}(G, w)$ sont donnés par les points d'intersection de $H_{1}(G, \mathbb{R})$ avec l'intérieur des faces de $B_{w, 1}$ de codimension plus grande ou égale à $b$ dans le cas où cette intersection est réduite à un point. Pour $C$ un circuit simple orienté, on note

$$
C=\sum_{i \in I(C)} \varepsilon_{i} e_{i}
$$

son développement dans la base des arêtes $\left\{e_{i}\right\}_{i=1}^{k}$ (ici, $\varepsilon_{i}= \pm 1$ ). Il est évident que le nombre $|I(C)|$ des arêtes dans (2.2) n'excède pas $k-b+1$. On considère la face $|I(C)|-1$ dimensionnelle $F(C)$ de $B_{w, 1}$ contenant les vecteurs

$$
\frac{\varepsilon_{i}}{w\left(e_{i}\right)} e_{i}, \quad i \in I(C)
$$

On voit facilement que

$$
X=\frac{1}{\|C\|_{w}} C=\frac{1}{\sum_{i \in I(C)} w\left(e_{i}\right)} \sum_{i \in I(C)} \varepsilon_{i} e_{i}
$$


est un point de $\operatorname{int}\left(\operatorname{conv}\left(\left\{\frac{\varepsilon_{i}}{w\left(e_{i}\right)} e_{i}, i \in I(C)\right\}\right)\right)$ où $\operatorname{conv}(A)$ désigne l'enveloppe convexe d'un ensemble fini de points $A$ et int $(B)$ désigne l'intérieur d'un ensemble $B$.

On montre alors que l'intersection $H_{1}(G, \mathbb{R}) \bigcap \operatorname{int}(F(C))$ est réduite à ce point. En effet, si $v$ est un autre point de $H_{1}(G, \mathbb{R}) \bigcap \operatorname{int}(F(C))$, les points $v$ et $X$ définissent un segment contenu dans l'intersection. Ceci implique qu'il existe des points d'intersection entre $H_{1}(G, \mathbb{R})$ et $F(C)$ dans un voisinage arbitraire de $X$. Autrement dit, il existe des points d'intersection (différents de $X$ ) appartenant à l'enveloppe convexe des $\left\{\frac{\varepsilon_{i}}{w\left(e_{i}\right)} e_{i}, i \in I(C)\right\}$. Si $u$ est un tel point, on peut écrire $u$ comme une combinaison linéaire des $\left\{\frac{\varepsilon_{i}}{w\left(e_{i}\right)} e_{i}, i \in I(C)\right\}$ dont les coefficents sont tous non nuls :

$$
u=\sum_{i \in I(C)} \alpha_{i} \frac{\varepsilon_{i}}{w\left(e_{i}\right)} e_{i} .
$$

On complète alors le vecteur $C$ en une base de $H_{1}(G, \mathbb{R})$ formée de circuits simples $\left\{C_{1}=C, C_{2}, \ldots, C_{b}\right\}$ de sorte que, pour chaque $i$ dans $\{1, \ldots, b\}$, il existe une arête $f_{i}$ contenue dans $C_{i}$ et n'appartenant pas aux $C_{j}$ pour $j \neq i$. L'analyse de la décomposition du vecteur $u$ dans cette base nous montre que le vecteur $u$ est nécessairement proportionnel à $C$. D'où une contradiction.

Maintenant, comme $\operatorname{dim}(F(C)) \leq k-b$, le vecteur $C /\|C\|_{w}$ est bien un sommet de $\mathcal{B}_{s t}(G, w)$. Ceci achève la démonstration.

Lemme A2 Soient $C_{1}$ et $C_{2}$ deux circuits simples orientés. Alors il existe des circuits simples orientés $\left\{D_{j}\right\}_{j \in J}$ (non uniquement définis) pour lesquels chaque arête n'est parcourue que dans un seul sens et tels que dans $H_{1}(G, \mathbb{R})$ :

$$
\left[C_{1}+C_{2}\right]=\sum_{j \in J}\left[D_{j}\right]
$$

Démonstration. On note $\left\{e_{i}\right\}_{i \in I}$ les arêtes de $G$ figurant dans $C_{1}$ et $C_{2}$ avec des directions opposées. On ôte les arêtes $\left\{e_{i}\right\}_{i \in I}$ de la reunion $C_{1} \cup C_{2}$. Notons $C$ la courbe obtenue. Elle possède une orientation induite par celles de $C_{1}$ et de $C_{2}$. En suivant cette orientation, $C$ se sépare naturellement en quelques courbes fermées et orientées $\left\{P_{l}\right\}_{l \in L}$. Cette représentation préserve la classe d'homologie :

$$
\left[C_{1}+C_{2}\right]=\sum_{l \in L}\left[P_{l}\right]
$$

Chaque courbe $P_{l}$ n'est pas, en général, un circuit simple car elle peut avoir des auto-intersections : des sommets, ou bien des arêtes parcourues plusieurs fois dans la même direction. On part d'un point d'auto-intersection et on parcourt $P_{l}$ en suivant l'orientation. En revenant au point de départ pour la première fois, nous coupons $P_{l}$ en deux courbes fermées et orientées. Chacune de ces deux nouvelles courbes a moins d'auto-intersections que la courbe initiale $P_{l}$. En répétant suffisamment ce procédé, nous obtenons un certain nombre de circuits simples qui engendrent la classe $\left[P_{l}\right]$. On applique ce procédé pour chaque $P_{l}, l \in L$. L'ensemble des circuits simples obtenus est noté $\left\{D_{j}\right\}_{j \in J}$. Pour achever la démonstration, il reste à remarquer que, par construction, les circuits simples orientés construits n'ont pas d'arête commune parcourue dans des directions opposées. 
Lemme A3 Pour chaque classe entière $a \in H_{1}(G, \mathbb{Z})$, il existe des circuits simples $\left\{C_{s}\right\}_{s \in S}$ (non uniquement définis) tels que

$$
a=\sum_{s \in S}\left[C_{s}\right] \quad \text { et }\|a\|_{w}=\sum_{s \in S}\left\|C_{s}\right\|_{w} .
$$

Démonstration. Comme les circuits simples orientés engendrent $H_{1}(G, \mathbb{Z})$, nous pouvons présenter une classe fixée $a \in H_{1}(G, \mathbb{Z})$ comme une somme de circuits simples orientés :

$$
a=\sum_{r \in R}\left[D_{r}\right] .
$$

Remarquons que la représentation (2.3) n'est pas uniquement définie et que certains circuits figurent, en général, plusieurs fois dans cette somme. En appliquant systématiquement le lemme $\mathbf{A} 2$ sur chaque paire de circuits $\left(D_{r_{1}}, D_{r_{2}}\right)$ ayant des arêtes en commun parcourues dans des directions opposées, nous arrivons au bout de ce procédé itératif sur une nouvelle représentation par des circuits $\left\{C_{s}\right\}_{s \in S}$ vérifiant le lemme.

Lemme A4 Soit $(G, w)$ un graphe pondéré dont la fonction poids $w$ est à valeurs rationnelles. Alors chaque sommet de $\mathcal{B}_{s t}(G, w)$ est proportionnel à un circuit simple orienté de $G$.

Démonstration. On considère un sommet $X$ de $\mathcal{B}_{s t}(G, w)$. On sait que c'est l'unique point d'intersection de $H_{1}(G, \mathbb{R})$ et d'une face de $B_{w, 1}$ de codimension plus grande ou égale à $b$. Comme les poids $\left\{w\left(e_{i}\right), 1 \leq i \leq k\right\}$ sont rationnels, les coordonnées des sommets de $B_{w, 1}$ sont rationnelles. Le sous-espace $H_{1}(G, \mathbb{R})$ est engendré par des vecteurs dont les coordonnées sont entières. Ceci implique que les coordonnées de $X$ sont rationnelles. On constate donc que $X=\lambda a$, où $a \in H_{1}(G, \mathbb{Z})$ est un vecteur indivisible et $\lambda$ est un facteur rationnel positif. On a également

$$
1=\|X\|_{w}=\lambda\|a\|_{w} .
$$

Décomposons $a$ en circuits simples orientés selon le lemme A3 :

$$
a=\sum_{s \in S}\left[C_{s}\right] .
$$

On tire alors du lemme $\mathbf{A} \mathbf{3}$ et de (2.4) l'égalité suivante:

$$
X=\frac{1}{\sum_{s \in S}\left\|C_{s}\right\|_{w}} \sum_{s \in S}\left(\left\|C_{s}\right\|_{w}\left(\frac{1}{\left\|C_{s}\right\|_{w}}\left[C_{s}\right] .\right)\right)
$$

D'après le lemme $\mathbf{A} \mathbf{1}$, les vecteurs $\left[C_{s}\right] /\left\|C_{s}\right\|_{w}$ pour $s \in S$ sont des sommets de $\mathcal{B}_{s t}(G, w)$ et $(2.5)$ implique à son tour que $X$ est un point de l'intérieur de l'enveloppe convexe de ces points. Comme $X$ est un sommet, on obtient donc que $S$ contient un seul circuit (la répétition d'un unique circuit n'étant pas possible, puisque $a$ a été choisi indivisible). La démonstration est achévée.

Les lemmes $\mathbf{A} \mathbf{1}$ et $\mathbf{A} \mathbf{4}$ impliquent le théorème $\mathbf{A}$ pour tout graphe pondéré dont la fonction poids est à valeurs rationnelles. D'autre part, ces lemmes montrent également que les directions des sommets de $\mathcal{B}_{s t}(G, w)$ ( $w$ étant à valeurs rationnelles) sont uniquement définies et ne dépendent que des circuit simples. Ceci, par continuité, implique le résultat pour des poids quelconques. 


\section{Polytope et classe conforme}

La démonstration du théorème va découler de plusieurs propositions techniques. On travaille dans la classe des métriques simpliciales. Ce sont des métriques lisses sur chaque simplexe d'une triangulation lisse avec une condition naturelle de coïncidence sur les faces communes. Pour plus de détails, le lecteur pourra consulter [1].

\subsection{Préparatifs}

Lemme B1 Soient $\left(X_{i}, h_{i}\right), i=1,2$ deux variétés munies de métriques simpliciales et $f: X_{1} \longrightarrow X_{2}$ une application simpliciale contractant les distances. On note $f_{*}$ l'application induite sur les homologies réelles unidimensionnelles. Alors

$$
f_{*}\left(\mathcal{B}_{s t}\left(h_{1}\right)\right) \subset \mathcal{B}_{s t}\left(h_{2}\right)
$$

Démonstration. Soit a un élément de $H_{1}\left(X_{1}, \mathbb{Z}\right)$ et supposons que $\gamma_{n}$ soit une courbe fermée lisse par morceaux réalisant la classe $n \mathbf{a}$. Comme f contracte les distances, on a $l_{h_{1}}\left(\gamma_{n}\right) \geq l_{h_{2}}\left(f\left(\gamma_{n}\right)\right)$, ce qui implique que

$$
\|\mathbf{a}\|_{h_{1}} \geq\left\|f_{*}(\mathbf{a})\right\|_{h_{2}} .
$$

Par continuité de la norme, l'inégalité (3.1) est valable pour toute classe réelle. On en déduit le résultat.

On note $\left\{s_{1}, \ldots, s_{k}\right\}$ un sous-ensemble de sommets de $K$ tels que

$$
K=\operatorname{conv}_{s}\left\{s_{1}, \ldots, s_{k}\right\}
$$

où $\operatorname{conv}_{s}$ désigne l'enveloppe convexe du symétrisé d'un ensemble. Les sommets de $K$ étant rationnels, on peut trouver pour tout $i=1 \ldots k$ un nombre $l_{i}>0$ tel que

$$
s_{i}=l_{i} \cdot \mathbf{v}_{i}
$$

où $\mathbf{v}_{i}$ est un vecteur entier indivisible. On se fixe $k$ courbes de $M$ lisses, disjointes, fermées et simples notées $\gamma_{1}, \ldots, \gamma_{k}$ telles que $\left[\gamma_{i}\right]=\mathbf{v}_{i}$ pour $i=1, \ldots, k$. On les suppose paramétrisées par longueur d'arc respectivement à la métrique donnée $g$.

Lemme B2 Dans $(M, g)$, il existe $k$ voisinages tubulaires disjoints $\left\{U_{i}\right\}_{i=1}^{k}$ des courbes $\left\{\gamma_{i}\right\}_{i=1}^{k}$ et une métrique $g_{1}$ lisse conforme à $g$ tels que

1. $l_{g_{1}}\left(\gamma_{i}\right)=l_{i}^{-1}, \quad 1 \leq i \leq k$;

2. les $U_{i}$ sont fibrés par des disques $g_{1}$-orthogonaux $\grave{a} \gamma_{i}$ et les projections $p_{i}: U_{i} \longrightarrow \gamma_{i}$ le long de ces disques contractent les $g_{1}$-distances.

Démonstration. On se fixe $\varepsilon>0$ suffisamment petit pour que les $\varepsilon$-voisinages tubulaires des courbes $\gamma_{i}$ respectivement à la métrique $g$ soient disjoints et qu'ils soient fibrés par des disques $g$-géodesiques orthogonaux aux $\gamma_{i}$. Notons $U_{i}$ ces voisinages tubulaires. On choisit une fonction $\lambda$ strictement positive sur $M$ qui soit constante égale à $l_{i} \cdot l_{g}\left(\gamma_{i}\right)^{-1}$ sur $U_{i}$. La metrique $\widehat{g}=\lambda^{2} g$ vérifie la propriété 1 . et les disques fibrant $U_{i}$ sont toujours géodesiques et ortogonaux aux $\gamma_{i}$ pour la 
métrique $\widehat{g}$. Puisque $\widehat{g}$ est conforme à $g$, et pour simplifier nos futures notations, nous pouvons supposer que la métrique de départ vérifie la propriété 1.

Soit $p_{i}: U_{i} \longrightarrow \gamma_{i}$ le fibré normale de $\gamma_{i}$. On considère un système de coordonnées semi-géodesique sur $U_{i}$ :

$$
\left(s, x_{2}, x_{3}, \ldots, x_{m}\right),
$$

ou $s=x_{1}$ est le paramétre naturel le long de $\gamma_{i}$ et les $\left(x_{2}, x_{3}, \ldots, x_{m}\right)=\bar{x}$ sont les coordonnées géodésiques sur les disques ortogonaux. Ce système est bien défini sur chaque ouvert du type $p_{i}^{-1}([s-\delta, s+\delta]$ pour $\delta>0$ suffisamment petit. Pour $q=(s, \bar{x}) \in U_{i}$, notons également $\mathbf{r}(q)=\operatorname{dist}\left(q, \gamma_{i}\right)$. Alors $\mathbf{r}^{2}$ est une fonction lisse définie sur $U_{i}$. Soient $\left\{g_{k l}(s, \bar{x})\right\}_{k, l=1}^{m}$ les coefficients de $g$ dans (3.2). Nous avons par construction

$$
g_{11}(s, \overline{0})=1 ; \quad g_{1 l}(s, \overline{0})=0, \quad 2 \leq l \leq m .
$$

Tout vecteur tangent $\mathbf{v} \in T_{q} U_{i}$ s'écrit dans la base $\left\{\frac{\partial}{\partial x_{k}}\right\}_{k=1}^{m}$

$$
\mathbf{v}=(\alpha, \bar{v}) ; \text { où } \bar{v}=\left(v_{2}, v_{3}, \ldots, v_{m}\right) \text {. }
$$

Nous pouvons calculer sa norme :

$$
\|\mathbf{v}\|_{g}^{2}=g_{11}(s, \bar{x}) \alpha^{2}+2 \alpha \sum_{k=2}^{m} g_{1 k}(s, \bar{x}) v_{k}+\|\bar{v}\|_{g^{\prime}}^{2},
$$

où $g^{\prime}$ est la restriction de $g$ à l'espace tangent au disque normal correpondant, sa matrice cö̈ncidant au bloc de $g$ dual à $g_{11}$.

Cherchons maintenant le facteur conforme défini sur $U_{i}$ sous la forme suivante :

$$
\lambda_{i}^{2}(q)=\frac{1+a \mathbf{r}(q)^{2}}{g_{11}(q)},
$$

où $a$ est une constante que nous allons choisir afin de vérifier la propriété 2 . du lemme. Sur chaque $U_{i}$, pour la métrique $g_{1}=\lambda_{i}^{2} g$, on tire de (3.4) que :

$$
\|\mathbf{v}\|_{g_{1}}^{2}-\alpha^{2}=\lambda_{i}^{2}\left(\frac{a g_{11}(s, \bar{x}) \mathbf{r}^{2}(s, \bar{x})}{1+a \mathbf{r}^{2}(s, \bar{x})} \alpha^{2}+2 \alpha \sum_{k=2}^{m} g_{1 k}(s, \bar{x}) v_{k}+\|\bar{v}\|_{g^{\prime}}^{2}\right) .
$$

Compte-tenu de (3.3), on a

$$
\left|\sum_{k=2}^{m} g_{1 k} v_{k}\right| \leq A \mathbf{r}(s, \bar{x})\|\bar{v}\|_{g^{\prime}}
$$

pour une constante $A>0$ appropriée. Nous obtenons alors de (6) l'inégalité suivante :

$$
\|\mathbf{v}\|_{g_{1}}^{2}-\alpha^{2} \geq \lambda_{i}^{2}\left(\frac{a g_{11} \mathbf{r}^{2}}{1+a \mathbf{r}^{2}} \alpha^{2}-2 A|\alpha| \mathbf{r}\|\bar{v}\|_{g^{\prime}}+\|\bar{v}\|_{g^{\prime}}^{2}\right) .
$$

Nous avons dans les parenthèses une forme quadratique en les arguments $|\alpha| \mathbf{r}$ et $\|\bar{v}\|_{g^{\prime}}$, qui est positive si

$$
\frac{a g_{11}}{1+a \mathbf{r}^{2}}>A^{2}
$$

Prenons $a=2 A^{2}$, alors cette inégalité est vérifiée pour $\mathbf{r}=0$ en accord avec (3.3). On en déduit par continuité qu'elle est vérifiée sur l'ouvert $U_{i}$ tout entier si l'on a 
choisi $\varepsilon$ suffisamment petit au départ. Finalement, pour le facteur conforme (3.5) avec $a=2 A^{2}$, on obtient de (3.7)

$$
\|\mathbf{v}\|_{g_{1}} \geq|\alpha|
$$

et la propriété 2 . est donc vérifiée.

Pour achever la démonstration, il reste à prolonger les facteurs locaux conformes $\left\{\lambda_{i}\right\}_{i=1}^{n}$ en une fonction lisse $\lambda$ strictement positive.

\subsection{Construction d'une métrique de référence}

Nous allons commencer par construire une métrique de référence sur $M$ pour laquelle la boule unité de la norme stable est bien le polytope demandé. Cette métrique n'appartient en général pas à la classe conforme de la métrique de départ.

Proposition B3 Il existe une métrique lisse $h$ sur $M$ tel que

1. $\left\|\frac{d \gamma_{i}(s)}{d s}\right\|_{h}=\left\|\frac{d \gamma_{i}(s)}{d s}\right\|_{g_{1}}$, où s est le paramètre $g_{1}$-naturel le long des $\gamma_{i}$;

2. $\mathcal{B}_{s t}(M, h)=K$.

Démonstration. La démonstration se déroule en deux étapes. Dans la première, on montre que l'on peut se ramener à construire une métrique simpliciale sur $M$ réalisant le polytope $K$ comme boule stable, et dans une seconde étape, on explicite la construction de cette métrique simpliciale.

Lemme B4 Supposons qu'il existe une métrique $\widehat{h}$ sur $M$ simpliciale, telle que : 1. $\mathcal{B}_{s t}(\widehat{h})=K$.

2. $\exists \varepsilon>0$ tels que la métrique $\widehat{h}$ soit lisse sur les $\varepsilon$-voisinages tubulaires $U_{\varepsilon}\left(\gamma_{i}\right)$ de $\gamma_{i}$ (qui sont disjoints).

3. les $\gamma_{i}$ sont des géodésiques minimisantes de longueur $l_{\widehat{h}}\left(\gamma_{i}\right)=\left|v_{i}\right|_{\text {st }}=l_{i}^{-1}$. Alors la proposition est vérifiée.

Démonstration du lemme. On se fixe une métrique lisse $h^{\prime}$ sur $M$, qui coïncide avec $\widehat{h}$ sur les $U_{\varepsilon}\left(\gamma_{i}\right)$. On peut choisir une fonction $\zeta$ lisse, telle que $\zeta=1$ sur $U_{\varepsilon / 3}\left(\gamma_{i}\right), \zeta=L$ sur $M \backslash \cup_{i=1}^{k} U_{2 \varepsilon / 3}\left(\gamma_{i}\right)$ et qui soit croissante sur chaque $U_{\varepsilon}\left(\gamma_{i}\right)$ respectivement à la coordonnée radiale. On choisit $L$ suffisamment grand pour que la métrique $h=\zeta . h^{\prime} \geq \widehat{h}$. Pour $i=1, \ldots, k, v_{i} \in \mathcal{B}_{s t}(h)$. On conclut alors en utilisant le lemme $\mathbf{B} 1$ et la convexité de la boule stable.

Nous allons maintenant construire une métrique simpliciale satisfaisant les hypothèses du lemme B4. Soit $\mathbb{T}^{b}=H_{1}(M, \mathbb{R}) / i\left(H_{1}(M, \mathbb{Z})\right)$ le jacobien de $M(i$ désigne l'inclusion canonique). On considère une métrique euclidienne $h_{0}$ sur $\mathbb{T}^{b}$ telle que pour toute courbe $\gamma$ telle que $[\gamma]=\sum_{i=1}^{k} \alpha_{i} . v_{i}$ où $\alpha_{i} \in \mathbb{N}$ pour tout $i=1, \ldots, k$, $l_{h_{0}}(\gamma) \geq \sum_{i=1}^{k}\left|\alpha_{i}\right| \cdot l_{i}^{-1}+3$. Fixons un point $x_{0}$ de $\mathbb{T}^{b}$. Soient $\vee_{i=1}^{k} C_{i}$ un bouquet de $\mathrm{k}$ cercles pointé en un point $x_{1}$. On forme un graphe $\Gamma$ en ajoutant à $x_{1}$ une arête notée $a$ dont une des extrémités est libre. Soit $h_{0}^{\prime}$ une métrique simpliciale sur $\Gamma$ telle que $l_{h_{0}^{\prime}}\left(C_{i}\right)=l_{i}^{-1}$ et $l_{h_{0}^{\prime}}(a)=1$. On fixe sur $\mathbb{T}^{b}$ une triangulation linéaire telle que $x_{0}$ soit un 0 -simplexe. On considère le complexe simplicial $\left(P_{1}, h_{1}\right)$ formé en recollant l'extrémité libre de $\left(\Gamma, h_{0}^{\prime}\right)$ à $\left(\mathbb{T}^{b}, h_{0}\right)$ en $x_{0}$ (voir figure 1 ).

On a une application évidente $f_{1}: P_{1} \rightarrow \mathbb{T}^{b}$ qui est l'identité sur $\mathbb{T}^{b}$ et qui envoie $C_{i}$ sur $c_{i}$ la géodésique minimisante réalisant $v_{i}$ basée en $x_{0}$ en dilatant les distances. 


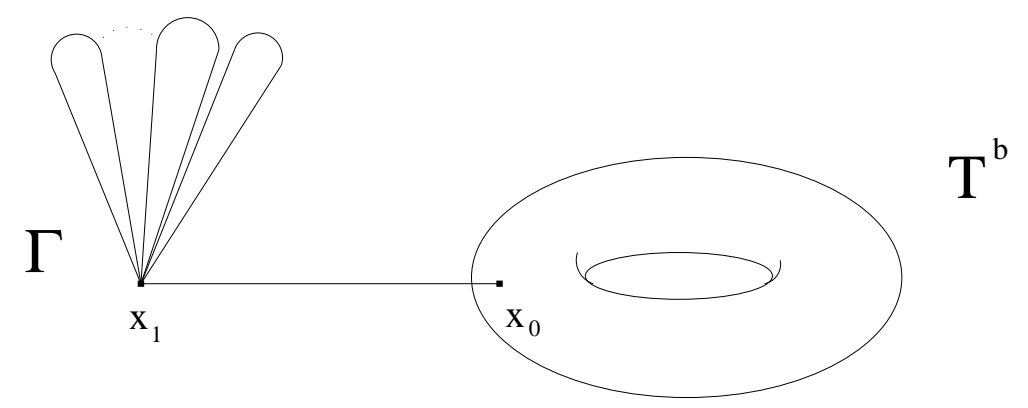

FIG. 1 - Le complexe $P_{1}$

Comme $f_{1}$ est l'identité sur $\mathbb{T}_{b}$, l'application induite en homologie $\left(f_{1}\right)_{*}: H_{1}\left(P_{1}, \mathbb{R}\right) \rightarrow H_{1}\left(\mathbb{T}^{b}, \mathbb{R}\right)$ est surjective. Pour $i=1, \ldots, k$, on réalise le cylindre de l'application $\left(f_{1}\right)_{\mid C_{i}}: C_{i} \rightarrow c_{i}$ de sorte que l'arête $a$ coïncide avec une génératrice du cylindre $Z_{i}$. On obtient ainsi un nouveau complexe simplicial $P_{2}$ dont $P_{1} \subset P_{2}$ est un sous-complexe. Remarquons également que $T^{b} \subset P_{2}$ est un rétract par déformation. On prolonge la métrique $h_{1}$ de $P_{1}$ à $P_{2}$ de la manière suivante. On note $q_{i}$ la longueur de la concaténation de $\tilde{C}_{i}=a \star C_{i} \star a^{-1}$ et $c_{i}$. Soit $p_{i}: S_{i}^{1} \rightarrow \tilde{C}_{i} \star c_{i}$ une application linéaire contractant deux fois les distances. Pour $i=1, \ldots, k$, on considère le cylindre de cette application, et on recolle une demi-sphère deux-dimensionnelle de rayon $q_{i} / \pi$ isométriquement le long de $S_{i}^{1}$ (qui est de longueur $2 q_{i}$ ). On note $h_{2}$ la métrique induite par notre construction.

Clairement,

$$
H_{1}\left(P_{2}, \mathbb{R}\right) \simeq H_{1}\left(\mathbb{T}^{b}, \mathbb{R}\right) .
$$

Remarquons que pour toute courbe lisse par morceaux $\gamma \subset P_{2}$, il existe une courbe $\gamma^{\prime} \subset P_{1}$ telle que $[\gamma]=\left[\gamma^{\prime}\right]$ et $l_{h_{2}}\left(\gamma^{\prime}\right) \leq l_{h_{2}}(\gamma)$.

\section{Lemme B5}

$$
\mathcal{B}_{s t}\left(h_{2}\right)=K
$$

Démonstration du lemme. Il est clair qu'une courbe minimisante de $\left(P_{2}, h_{2}\right)$ est contenue dans $P_{1}$. On se fixe une classe entière $v$ appartenant au sous-réseau $\Delta_{k}$ engendré par $\left\{v_{i}\right\}_{i=1}^{k}$. Etant donné $n \geq 1$, on choisit $\beta_{n}$ une courbe réalisant n.v. Quitte à modifier notre courbe $\beta_{n}$, on peut supposer que la courbe est obtenue comme la concaténation de deux courbes : la première $\beta_{n, 1}$ est contenue dans $\mathbb{T}^{b}$ et la seconde $\beta_{n, 2}$ dans $\Gamma$. La classe $v$ appartenant au sous-réseau $\Delta_{k}$, et de la structure de l'homologie réelle unidimensionnelle de $\Gamma$, on déduit que $v$ et $\left[\beta_{n, 2}\right] \in \Delta_{k}$ et donc que $\left[\beta_{n, 1}\right] \in \Delta_{k}$. On écrit $\left[\beta_{n, 1}\right]=\sum_{i=1}^{k} \alpha_{i} .\left[C_{i}\right]$ et comme $l_{h_{0}}\left(\beta_{n, 1}\right) \geq \sum_{i=1}^{k}\left|\alpha_{i}\right| \cdot l_{i}^{-1}+3$, on peut diminuer la longueur de $\beta_{n}$ en remplaçant la partie $\beta_{n, 1}$ par la courbe $a \star \prod_{i=1}^{k} C_{i}^{\alpha_{i}} \star a^{-1}$. Ceci entraîne que toute courbe minimisante est contenue dans $\Gamma$. Donc $|v|_{s t}=\min \left\{\sum_{i=1}^{k}\left|\alpha_{i}\right| \cdot l_{i}^{-1} \mid v=\sum_{i=1}^{k} \alpha_{i} \cdot v_{i}\right\}$ pour tout $v$ dans le sous-réseau engendré par les $\left\{v_{i}\right\}_{i=1}^{k}$. Par continuité de la norme et du fait que $\operatorname{rang}\left(\Delta_{k}\right)=b$ (comme $\operatorname{int}(K) \neq \emptyset$ ), on en déduit le résultat.

On peut remplacer dans notre complexe simplicial $P_{2}$ les cercles $C_{i}$ pour $i=$ 
$1, \ldots, k$ par des tubes $m$-dimensionnels $C_{i} \times D^{m-1}$ ou $C_{i} \widehat{\times} D^{m-1}$ (selon l'orientabilité des $U_{i}$ ), munis d'une métrique produit (euclidienne sur $D^{m-1}$ ) dont la projection radiale contracte les distances et telle que les disques 2-dimensionnels rattachés le soient le long d'un cercle du bord de ce tube. On note à nouveau $\left(P_{2}, h_{2}\right)$ le complexe simplicial obtenu. Cette opération ne modifie pas la forme de la boule stable.

Soit $\mathcal{J}: M \rightarrow \mathbb{T}^{b} \subset P_{2}$. Etant donnés les $k$ voisinages tubulaires topologiques disjoints $U_{i}$ de $\gamma_{i}$ pour $i=1, \ldots, k$, on modifie $\mathcal{J}$ dans sa classe d'homotopie de sorte que $\mathcal{J}\left(\gamma_{i}\right)=C_{i}$ et que $\mathcal{J}\left(U_{i}\right)=C_{i} \times D^{m-1}$ ou $C_{i} \widehat{\times} D^{m-1}$.

On peut alors approximer $\mathcal{J}$ par une application $\mathcal{J}_{1}$ qui soit simpliciale sur $M$ et qui coïncide avec $\mathcal{J}$ dans chaque $U_{i}$ pour $i=1, \ldots, k$ (voir [13]). La métrique $\mathcal{J}_{1}^{*}\left(h_{2}\right)$ tirée en arrière peut être dégénérée en-dehors des voisinages tubulaires. On se fixe une métrique lisse quelconque $h_{3}$ sur $M$ et $\xi$ une fonction lisse et croissante d'argument $q$ vérifiant

$$
\xi(q)=\left\{\begin{array}{lll}
0 & \text { si } & q \in \cup_{i=1}^{k} U_{i}\left(\frac{2 \varepsilon}{3}\right) \\
1 & \text { si } & q \in M \backslash \cup_{i=1}^{k} U_{i}(\varepsilon) .
\end{array}\right.
$$

Alors $\mathcal{J}_{1}:\left(M, \mathcal{J}_{1}^{*}\left(h_{2}\right)+\xi h_{3}\right) \rightarrow\left(P_{2}, h_{2}\right)$ contracte les distances et la métrique ainsi construite vérifie les propriétés annoncées.

\subsection{Démonstration du théorème $B$}

Sur la variété initiale $(M, g)$, on considère maintenant les objets construits dans le lemme $\mathbf{B 2}$ et la proposition ci-dessus. On choisit deux fonction lisses et croissantes d'argument $t$ vérifiant

$$
\phi(t)= \begin{cases}0 & \text { si } t \leq \frac{\varepsilon}{3} \\ t & \text { si } \frac{2 \varepsilon}{3} \leq t\end{cases}
$$

et

$$
\psi(t)= \begin{cases}0 & \text { si } t \leq \frac{\varepsilon}{9} \\ 1 & \text { si } \frac{2 \varepsilon}{9} \leq t\end{cases}
$$

où $\varepsilon$ est le rayon des voisinages tubulaires $\left\{U_{i}\right\}_{i=1}^{k}$ du lemme B2. On considère un point $q \in U_{i}$ et $\mathbf{r}(q)$ la fonction radiale définie ci-dessus. Soit $\eta_{q}(s)$ l'unique géodesique locale passant par $q$, orthogonale à $\gamma_{i}$ et telle que $\eta_{q}(0) \in \gamma_{i}$. On définit les applications

$$
G_{i}: U_{i} \longrightarrow U_{i} ; 1 \leq i \leq k
$$

en posant $G_{i}(q)=\eta_{q}(\phi(\mathbf{r}(q)))$. Remarquons que $G_{i}(q)=q$ si $\mathbf{r}(q) \geq \frac{2 \varepsilon}{3}$ et que $G_{i}(q)=\eta_{q}(0) \mathbf{r}(q) \leq \frac{\varepsilon}{3}$. Les applications locales $\left\{G_{i}\right\}_{i=1}^{k}$ se prolongent en une application

$$
G: M \longrightarrow M \text {. }
$$

Remarquons que $G$ induit en homologie l'application identité.

On définit maintenant les fonctions locales suivantes

$$
\Psi_{i}: U_{i} \longrightarrow \mathbb{R} ; 1 \leq i \leq k
$$

en posant $\left.\Psi_{i}(q)=\psi(\mathbf{r}(q))\right)$. Remarquons que $\Psi_{i}(q)=1$ si $\mathbf{r}(q) \geq \frac{2 \varepsilon}{9}$ et que $\Psi_{i}(q)=0$ si $\mathbf{r}(q) \leq \frac{\varepsilon}{9}$. Ces fonctions locales $\left\{\Psi_{i}\right\}_{i=1}^{k}$ se prolongent en une fonction

$$
\Psi: M \longrightarrow \mathbb{R}
$$


Choisissons finalement $B>0$ tel que

$$
g^{\prime}=(1+B \Psi) g_{1} \geq g_{1}+\Psi G_{*}(h) .
$$

On voit facilement par construction et de la propriété 2. de la proposition B3 que $G:\left(M, g^{\prime}\right) \rightarrow(M, h)$ contracte les distances. Du lemme B1, on tire

$$
\mathcal{B}_{s t}\left(g^{\prime}\right) \subset \mathcal{B}_{s t}(h)=K
$$

Par construction, les métrique $g^{\prime}$ et $g_{1}$ coïncident dans les $\frac{\varepsilon}{9}$-voisinages tubulaires des $\left\{\gamma_{i}\right\}_{i=1}^{n}$. Ceci avec la propriété 1 . du lemme B2 implique que les sommets de $K$ appartiennent à $\mathcal{B}_{s t}\left(g^{\prime}\right)$. La convexité nous donne alors le résultat.

\section{Références}

[1] Babenko, I. : Souplesse intersystolique forte des variétés fermées et des polyèdres, Ann. Inst. Fourier (Grenoble) 52 (2002) no. 4, 1259-1284.

[2] Bacher, R., de la Harpe, P. \& Nagnibeda T. : Lattices defined by finite graphs, Bull. Soc. Math. France 125 (1997), 167-198.

[3] Bangert, V. : Minimal geodesics, Ergod. Th. \& Dynam. Sys. 10 (1989), 263286.

[4] Bangert, V. : Geodesics rays, Busemann functions and monotone twist maps, Calc. Var. 2 (1994), 49-63.

[5] Burago, D., Ivanov, S. \& Kleiner, B. : On the structure of the stable norm of periodic metrics, Math. Res. Let. 4 (1997), 791-808.

[6] Federer, H. : Real flat chains, cochains and variational problems, Indiana Univ. Math. J. 24 (1974), 351-407.

[7] Gromov, M., Lafontaine, J. \& Pansu, P. : Structures métriques pour les variétés riemanniennes, CEDIC, Paris (1981).

[8] Hedlund, G.A. : Geodesics on a two-dimensional Riemannian manifold with periodic coefficients, Ann. of Math. 33 (1932), 719-739.

[9] Massart, D. : Stable norm of surfaces : local structure of the unit ball at rationnal directions, Geom. Funct. Anal. 7 (1997), 996-1010.

[10] Massart, D. : Normes stables des surfaces, thèse de doctorat, Ecole Normale Supérieure de Lyon (1996).

[11] Massart, D. : On Aubry sets and Mather's action functional, Isr. J. Math. 134 (2003), 157-171.

[12] Morse, M. : A fundamental class of geodesics on any closed surface of genus greater than one, Trans. Amer. Soc. 26 (1924), 25-60.

[13] Spanier, E. : Algebraic Topology, McGrow-Hill Book Company (1966). 